\title{
PARÁMETROS FISICOQUÍMICOS, MICROBIOLÓGICOS Y SENSORIALES DE SALCHICHAS ELABORADAS CON INCLUSION DE QUITOSANO
}

\section{PHYSICOCHEMICAL, MICROBIOLOGICAL AND SENSORY PARAMETERS OF SAUSAGES PREPARED WITH INCLUSION OF CHITOSAN}

\author{
José Igor Hleap ${ }^{1}$, Lina Cardona ${ }^{2}$, Johana Agudelo², Alejandra Gómez ${ }^{2}$
}

\begin{abstract}
${ }^{1}$ Ingeniero Pesquero, PhD. Ingeniería de Alimentos, Docente. Universidad Nacional de Colombia, Facultad de Ingeniería y Administración, Palmira, Valle del Cauca, Colombia, e-mail: jihleapz@unal.edu.co; ${ }^{2}$ Estudiantes de Ingeniería Agroindustrial. Universidad Nacional de Colombia, Facultad de Ingeniería y Administración, Palmira, Valle del Cauca, Colombia, e-mail: Imcardonas@unal.edu.co, djagudeloc@unal.edu.co, maagomezhe@unal.edu.co
\end{abstract}

Rev. U.D.C.A Act. \& Div. Cient. 18(2): 455-464, Junio-Diciembre, 2015

\section{RESUMEN}

Las industrias cárnica y pesquera están buscando constantemente ingredientes, como alternativas seguras para aumentar las características de sabor y de color de los productos finales, sin afectar las propiedades sensoriales, nutricionales y funcionales. En este estudio, se evaluó el quitosano, como un elemento extensor en embutidos de carne y de pescado. Se elaboraron cuatro tipos de embutidos: dos, a base de carne de cerdo y dos, de filete de tilapia. Cada grupo, se dividió en dos subgrupos. En el primer subgrupo, se añadió harina de trigo, como único componente ( $100 \%$ de harina de trigo), mientras que en el otro, se añadieron $35 \%$ quitosano y $65 \%$ de harina de trigo. Se sometieron a análisis microbiológicos, fisicoquímicos y sensoriales; el análisis sensorial fue realizado por un grupo de 77 panelistas no entrenados, utilizando una escala hedónica de siete puntos, estudiando el sabor, el color, el olor, la textura y la opinión general; todas las características fueron aceptables, excepto el color. Los parámetros fisicoquímicos no mostraron diferencias estadísticamente significativas dentro de cada subgrupo de salchichas; sin embargo, la comparación entre los dos grupos, mostró diferencias significativas $(p<0,05)$ en el contenido de proteína y de grasa. Para las salchichas de carne de cerdo, más del $50 \%$ de los panelistas juzgaron positivamente sobre los parámetros sensoriales. A partir de los productos elaborados, las salchichas de carne de cerdo tuvieron mejor aceptación organoléptica, para las de filete de pescado no fue así, probablemente, debido a la falta de conocimiento de este producto no tradicional.

Palabras clave: Embutidos, evaluación sensorial, industria cárnica, industria pesquera, sustancias extensoras.

\section{SUMMARY}

Meat and fish industries are constantly searching ingredients as safe alternatives to increase flavor and color characteristics of the final products without affecting sensory, nutritional and functional properties. In this study, chitosan was evaluated as an extender element in meat and fish sausages. Four types of sausages were produced: two based on pork meat and two based on tilapia fillet. Each group was divided into two more subgroups. In the first subgroup, wheat flour was added as the only component (100\% of wheat flour) while in the other subgroup $35 \%$ chitosan and $65 \%$ wheat flour were added. They were submitted to microbiological, physicochemical and sensory analysis. Sensory analysis was made by a group of 77 untrained panelists, using a seven-point hedonic scale, analyzing the taste, color, smell, texture and general opinion attributes. All the characteristics were acceptable except color. The physicochemical parameters showed no statistically significant differences within each group of sausages. However, comparison between groups of sausages, showed significant differences $(p<0.05)$ in protein and fat contents. For the pork meat sausage, more than $50 \%$ of the panelists judged positively about sensory parameters. From the processed products, the pork sausages meat had the best organoleptic acceptance, while the fish fillet sausages had not well acceptance, probably due to the absence of knowledge of this non-traditional product.

Key words: Sausages, sensory evaluation, meat industry, fish industry, extensor substances. 


\section{INTRODUCCIÓN}

La actividad pesquera en Colombia, se caracteriza por el manejo, a nivel de pequeñas empresas, de un número importante de recursos de origen hidrobiológico. Entre estos recursos, se dispone de los camarones, cuyo destino es el mercado internacional. Según datos de la AUNAP, la captura de estos recursos naturales en Colombia está representada en 3.790t anuales (AUNAP, 2014). Para las exportaciones, se usan las colas enteras, desvenadas y congeladas, quedando, como subproducto de esta operación, los caparazones, que contienen, aproximadamente, entre el $20-30 \%$ de un polímero natural, la quitina (Ramya et al. 2012), de la cual, extrae el quitosano, que tiene aplicaciones en las múltiples industrias (Mármol et al. 2011).

El quitosano es uno de los pocos polisacáridos catiónicos naturales, con un pKa de alrededor de 6,5, lo que le confiere propiedades muy particulares (Krajewska, 2004); se deriva de la quitina, mediante la desacetilación de la misma (Rabea et al. 2003). Presenta la habilidad de ligar lípidos y metales y puede extender la vida de anaquel de productos alimenticios frescos y con alta actividad de agua, como frutas, verduras y carnes (Jeon et al. 2002).

La quitina, sustancia de la cual se obtiene el quitosano, es el segundo polisacárido en abundancia en la naturaleza, después de la celulosa, derivado del exoesqueleto de los crustáceos, así como también, de las paredes celulares de hongos e insectos (Tharanathan \& Kittur, 2003), aspecto que hace que en el mercado actual tenga un potencial de aceptación, como ingrediente en la elaboración de múltiples productos, entre otros, alimenticios (Mahdy et al. 2013). La principal fuente comercial la constituyen los desechos de camarón y de cangrejo (Rinaudo, 2006).

La quitina y el quitosano tienen un gran impacto económico, debido a sus actividades biológicas y sus aplicaciones técnicas, industriales y biomédicas. Gracias a las propiedades del quitosano, como la adsorción, las propiedades formadoras de película (Velázquez-Moreira \& Guerrero, 2014) y las propiedades antioxidantes (Georgantelis et al. 2007) y antimicrobianas (Zhang et al. 2011), se utiliza en la preservación de alimentos (No et al. 2007), en la elaboración de cosméticos (Abdel-Mohsen et al. 2014), en la agricultura (Zeng et al. 2012), en la biotecnología (Mármol et al. 2011), en el procesamiento de fibras textiles (Abdel-Mohsen et al. 2012), así como en los campos de la medicina (Przekora \& Ginalska, 2014).

Tradicionalmente y con la finalidad de reducir los costos de producción en la formulación de los productos cárnicos, se han introducido algunas sustancias, denominadas "extensores", cuyo objetivo es sustituir una parte de la carne, ofre- ciendo el aporte proteico y funcional adecuado (Andújar et al. 2000). Los ligadores, al igual que los extensores, buscan aumentar los valores nutricionales y funcionales de los productos finales; generalmente, se basan en harinas, féculas o almidones. Son materiales, que sólo ocupan el lugar de la carne, ligando una cantidad de agua considerable, pero sin un aporte proteico. Estos extensores cárnicos tienen propiedades funcionales, tales como la retención de agua, la emulsificación de grasas y la gelificación, importantes desde el punto de vista tecnológico (Güemes, 2007). Así, la presencia de un tipo de extensor u otro, no solo dará economía a la fórmula sino que, también, actuará como facilitador del proceso (Blanno, 2006).

En la industria alimentaria, la quitina y el quitosano tienen usos, como aditivos en los alimentos, con carácter espesante, gelificante y emulsificante (Shahidi et al. 1999; Rinaudo, 2006).

Este trabajo buscó aplicar el quitosano como extensor, en productos emulsionados, derivados de la carne y del pescado y evaluar, sensorial, microbiológica y bromatológicamente, las características de los productos finales.

\section{MATERIALES Y MÉTODOS}

Muestras de estudio. Los caparazones de camarón (Penaeus vannamei) fueron traídos desde el puerto de Buenaventura, Valle del Cauca, Colombia, al Laboratorio de Tecnología de Carnes de la Universidad Nacional de Colombia -sede Palmira. La carne de cerdo, al igual que los filetes de tilapia y el rebanado o grasa dorsal fueron adquiridos en un supermercado de la ciudad de Palmira, Valle del Cauca; los demás insumos y aditivos, se obtuvieron en una casa comercializadora de insumos para la industria alimenticia.

A partir de los caparazones del camarón, se obtuvo la quitina, según el procedimiento planteado por Shahidi et al. (1999), el cual, se desarrolló en Laboratorio de Química de la Universidad Nacional de Colombia - sede Palmira. Por desacetilación de la quitina, utilizando una solución de $\mathrm{NaOH}$ al $50 \%(\mathrm{p} / \mathrm{v})$ y una temperatura de $40^{\circ} \mathrm{C}$, durante 15 horas, en una relación 1:4, se obtuvo el quitosano.

Se elaboraron dos tipos de salchichas: una, a base de carne de cerdo (Sus scrofa domestica) y, la otra, de filetes de tilapia (Oreochromis sp.) (Tabla 1). Se trabajó con dos matrices cárnicas de diferentes características funcionales y sensoriales, ya que se buscaba evaluar el comportamiento de algunos parámetros fisicoquímicos, microbiológicos y sensoriales, de dichos productos, con adición de quitosano, como elemento extensor, independiente del tipo de materia prima cárnica utilizada. En ambos casos, se desarrollaron dos alternativas: una, con el $100 \%$ de la cantidad de harina de trigo estipulada 
por la formulación, desarrollada en experimentaciones previas y, la otra, sustituyendo el $35 \%$ de ésta por el quitosano elaborado. Con base en las experimentaciones preliminares, se determinó que un mayor porcentaje de adición de quitosano conlleva a la alteración de las características texturales del producto final, afectando parámetros, como la adhesividad, la cohesividad y la masticación; por el contrario, un menor porcentaje de adición del quitosano, no se refleja en las características de ligazón, que se buscan con la adición de extensores no cárnicos.

Las materias primas, insumos y aditivos, se pesaron de acuerdo a la formulación. Se trabajaron por separado las salchichas de carne de cerdo y las de filete de tilapia. Después de descongelar las materias primas cárnicas, se procedió a fraccionarlas manualmente en trozos, cerca de $5 \mathrm{~cm}$ de lado, con el fin de facilitar las operaciones de mezclado, en un cúter Hobart 84181-D Corporation Troy, Ohio, USA, durante 15 minutos, hasta obtener una emulsión homogénea. Se embutió en una embutidora hidráulica marca Javar EM 30 Javar Tecnología Alimentaria Bogotá, Colombia. Se utilizó tripa artificial Amicel $^{\circledR}$ calibre 22. Las salchichas, se amarra- ron manualmente, de acuerdo a un peso de $60 \mathrm{~g}$ por unidad. Para lograr la coagulación de las proteínas y la consecuente cocción del embutido, las salchichas se sometieron al proceso de escaldado en agua a $80^{\circ} \mathrm{C}$, hasta que alcanzaron una temperatura interna de $72^{\circ} \mathrm{C}$, que se verificó con un termómetro de aguja Checktemp HI 98501 Hanna Instruments Ltda., Inglaterra, introducido en el centro de una salchicha, tomada en forma aleatoria. Con el objetivo de reducir la temperatura de las salchichas, se realizó una inmersión en agua fría, para generar un choque térmico del producto, que permaneció, aproximadamente, a $15^{\circ} \mathrm{C}$, durante un tiempo de $5 \mathrm{~min}$. El producto terminado, se empacó en bolsas especiales con una máquina al vacío EGARVAC Basic B. Vacarisses, Barcelona, España, en presentaciones de siete salchichas, con un rótulo, identificando el tipo de salchicha. Las salchichas empacadas, se almacenaron a temperaturas de refrigeración $2^{\circ} \mathrm{C} \pm 2^{\circ} \mathrm{C}$, hasta el momento de realizar las pruebas microbiológicas, fisicoquímicas y sensoriales. Los análisis microbiológicos y fisicoquímicos, se hicieron por triplicado y fueron contratados con un laboratorio comercial de análisis de alimentos, ubicado en la ciudad de Cali, Valle del Cauca.

Tabla 1. Formulación utilizada para la elaboración de las salchichas.

\begin{tabular}{|c|c|c|c|c|c|}
\hline \multirow{3}{*}{ Producto } & \multicolumn{2}{|c|}{ Salchichas de carne de cerdo } & \multicolumn{2}{|c|}{ Salchichas de filete de tilapia } & \\
\hline & $100 \%$ harina de trigo & $\begin{array}{c}65 \% \text { harina de trigo y } \\
35 \% \text { quitosano }\end{array}$ & $\begin{array}{l}100 \% \text { harina } \\
\text { de trigo }\end{array}$ & $\begin{array}{c}65 \% \text { harina de trigo } \\
\text { y } 35 \% \text { quitosano }\end{array}$ & \\
\hline & $g$ & g & $g$ & $g$ & $\%$ \\
\hline Carne de cerdo (pH 6,5) & 638,30 & 638,30 & - & - & \multirow{2}{*}{38,91} \\
\hline Filete de tilapia & - & - & 638,30 & 638,30 & \\
\hline Rebanado (grasa dorsal) & 279,80 & 279,80 & 279,80 & 279,80 & 17,05 \\
\hline Ligador XT-202 & 42,20 & 42,20 & 42,20 & 42,20 & 2,57 \\
\hline Hielo & 474,00 & 474,00 & 474,00 & 474,00 & 28,89 \\
\hline Sal & 30,00 & 30,00 & 30,00 & 30,00 & 1,82 \\
\hline Nitral & 7,10 & 7,10 & 7,10 & 7,10 & 0,43 \\
\hline Condimento salchicha & 19,00 & 19,00 & 19,00 & 19,00 & 1,15 \\
\hline Glutamato mono sódico & 2,20 & 2,20 & 2,20 & 2,20 & 0,13 \\
\hline Polifosfatos & 7,90 & 7,90 & 7,90 & 7,90 & 0,48 \\
\hline Ascorbato & 4,70 & 4,70 & 4,70 & 4,70 & 0,28 \\
\hline Humo líquido & 4,70 & 4,70 & 4,70 & 4,70 & 0,28 \\
\hline Cebolla en polvo & 4,70 & 4,70 & 4,70 & 4,70 & 0,28 \\
\hline Ajo en polvo & 4,70 & 4,70 & 4,70 & 4,70 & 0,28 \\
\hline Harina de trigo & 121,00 & 78,65 & 121,00 & 78,65 & \multirow{2}{*}{7,37} \\
\hline Quitosano en polvo & - & 42,35 & - & 42,35 & \\
\hline TOTAL & 1640,30 & 1640,30 & 1640,30 & 1640,30 & 100,00 \\
\hline
\end{tabular}

Fuente: Adaptado de Hleap \& Velasco, 2012. 
Análisis microbiológico. Para cada uno de los cuatro productos elaborados, se determinó: Aerobios mesófilos totales UFC g ${ }^{-1}$, según norma NTC 4519 (2009); Coliformes totales UFC $\mathrm{g}^{-1}$, según norma NTC 4458 (2007); Escherichia coli UFC $g^{-1}$, según norma NTC 4458 (2007); Esporas de Clostridium Sulfito Reductor UFC $\mathrm{g}^{-1}$, según norma INVIMA (1998); Salmonella en 25g, según norma NTC 4574 (2007) y Estafilococo coagulasa positiva UFC $\mathrm{g}^{-1}$, según norma NTC 4779 (2007).

Análisis fisicoquímico. Se realizaron los siguientes análisis: humedad y materia volátil, según el método de secado directo (AOAC 950.46, 2011); proteína total medida como $\mathrm{N}$ x 6,25, según el método Kjeldhal (AOAC 2011.04, 2011); grasa total, según el método extracto etéreo (AOAC 991.36, 2011); cenizas, de acuerdo al método (AOAC 991.36, 2011); carbohidratos totales, por método de cálculo; valor energético, medido como calorías totales en $\mathrm{Kcal} / 100 \mathrm{~g}$, según el método de la bomba calorimétrica de Berthelot - Mahler; nitritos en partes por millón, según el método químico (AOAC 973.31, 2011) y presencia de almidón \%, según el método colorimétrico.

Análisis sensorial. Se realizaron pruebas afectivas, para lo cual, se contó con el concurso de 77 jueces evaluadores no entrenados. Para realizar las degustaciones, se calentaron las salchichas a la plancha, a una temperatura aproximada de $90^{\circ} \mathrm{C}$ y se trocearon a un tamaño promedio de 1 a $2 \mathrm{~cm}$. La degustación, se realizó entre las 9 y 11 a.m. y las 2 y 5 p.m., para evitar que los jueces tuvieran sesgos, debidos a la saciedad o al hambre. Se ofreció a cada juez evaluador un trozo de cada uno de los productos, identificado con un número aleatorio de tres cifras; entre degustación y degustación transcurrieron, aproximadamente, tres minutos, tiempo en el que los evaluadores debieron enjuagar la boca con agua, para evitar sesgos sensoriales, entre una muestra y la siguiente. Las pruebas realizadas fueron: prueba de grado de satisfacción y prueba de aceptación y preferencia. Para la primera, los jueces, después de probar cada una de las salchichas, deberían diligenciar una encuesta, con una escala hedónica de siete puntos (Tabla 2), que indicarían el grado de satisfacción del consumidor, para los atributos sabor, olor, color, textura y opinión general. La segunda prueba, se estableció en orden aleatorio y se llevó a cabo, simultáneamente, con la prueba de grado de satisfacción. Los jueces evaluadores deberían escoger la de mayor agrado sensorial entre las dos muestras, con inclusión de $35 \%$ de quitosano, ya que la adición de esta sustancia era lo que se quería evaluar en el producto final, además, que permitió obtener datos sobre las características de los jueces evaluadores.

Análisis estadístico. Para procesar la información recolectada en las encuestas, se utilizó el programa estadístico IBM SPSS Statistics 19, donde se obtuvo un análisis de medias y varianza ANOVA y la prueba Tukey, para determinar las diferencias estadísticas entre las muestras.

\section{RESULTADOS Y DISCUSIÓN}

Parámetros fisicoquímicos. El análisis de los parámetros fisicoquímicos evaluados (Tabla 3) presentó diferencias significativas $(p<0,001)$, para humedad, proteína, grasas, carbohidratos, valor energético y contenido de nitritos. Las salchichas elaboradas a base de filetes de tilapia, estadísticamente, presentan los mayores valores de humedad, de cenizas y de carbohidratos y, los más bajos contenidos, de proteína y de grasas. Los valores de humedad, de proteína y de carbohidratos, para las salchichas fabricadas a base de filete de tilapia, son similares a los mostrados por Hleap \& Velasco (2010a); sin embargo, para las grasas el valor obtenido fue menor, debido al bajo porcentaje de inclusión de grasa de cerdo en los productos elaborados. Comparando los dos tipos de salchichas, se puede observar que el contenido de humedad es similar, ajustándose ambos a lo estipulado por la Norma Técnica Colombiana NTC 1325 (ICONTEC, 2008), que establece, para productos denominados Calidad Premium,

Tabla 2. Escala hedónica utilizada en la prueba del grado de satisfacción.

\begin{tabular}{|c|c|}
\hline Calificación hedónica & $\begin{array}{c}\text { Calificación } \\
\text { numérica }\end{array}$ \\
\hline Me gusta muchísimo & 7 \\
\hline Me gusta mucho & 6 \\
\hline Me gusta ligeramente & 5 \\
\hline Ni me gusta ni me disgusta & 4 \\
\hline Me disgusta ligeramente & 3 \\
\hline Me disgusta mucho & 2 \\
\hline Me disgusta muchísimo & 1 \\
\hline
\end{tabular}


el contenido máximo de humedad, de $67 \%$. El contenido de humedad obtenido para las salchichas a base de carne de cerdo fue similar al encontrado por O'Flynn et al. (2014), de $63,06 \%$; en otro estudio realizado con salchichas fabricadas a base de bagre tailandés (Clarias macrocephalus), por Intarasirisawat et al. (2014), se registraron valores ligeramente superiores, de $75 \%$, a los hallados en este trabajo, utilizando harina de tapioca (Manihot esculenta), como agente extensor y, finalmente, Yang et al. (2007) obtuvieron 67 a $68 \%$ de humedad en salchichas de cerdo bajas en grasa, con adición de harina de avena, como sustancia extensora. El contenido de humedad de las salchichas, se encuentra estrechamente relacionado con el tipo de ingrediente cárnico utilizado para su elaboración, lo cual, puede ser clasificado de acuerdo a su capacidad de retención de agua (Savadkoohi et al. 2014). Probablemente, los valores de humedad hallados, se pueden deber a que la carne de cerdo, los filetes de tilapia y otras matrices cárnicas utilizadas, se caracterizan por una disminución de la capacidad de retención de humedad, durante el escurrido, el secado y el almacenamiento de los productos finales, así como a las diferentes formulaciones empleadas en su fabricación, que son muy variadas, dependiendo de los gustos sensoriales en diferentes países y de las normatividades vigentes en los mismos.

Para los dos tipos de salchichas, se hallaron valores de proteína similares, no encontrándose diferencias estadísticas. La diferencia presentada, se puede deber a la mayor pérdida de humedad en las salchichas de pescado, durante el escal- dado, ya que el músculo es una carne con menor contenido de tejido conectivo y fibroso y, por lo tanto, es más sensible a estos procesos térmicos. Los valores obtenidos para las salchichas de carne de cerdo son superiores, a los reportados en salchichas tipo Viena, por De Landaeta et al. (2012), quienes encontraron valores de 11,3 a 11,8\%. Para las salchichas de filete de tilapia, los valores obtenidos son superiores a los reportados por Hleap et al. (2010b), quienes presentaron valores de 10,10\%, para salchichas elaboradas a partir de carduma (Cetengraulis mysticetus) y plumuda (Opsithonema spp.), con adición de harina de trigo, como extensor. En contraste, están por debajo de los valores obtenidos en otro trabajo -en salchichas elaboradas con carne de atún y res-, de $15,53 \%$, en donde se utilizó, igualmente, harina de trigo, como sustancia extensora (García et al. 2005).

El contenido de grasa de las salchichas de los dos grupos mostraron diferencias significativas $(p<0,05)$, atribuibles a las variaciones en el contenido de grasa de las materias primas utilizadas; sin embargo, dentro de cada grupo de salchichas, la adición de quitosano, como sustancia extensora, no influyó en el contenido de grasa en los productos finales, ya que no se observaron diferencias significativas entre los valores obtenidos, lo cual, se explica por ser el quitosano un polisacárido libre de sustancias lipídicas. Estos datos difieren de los presentados por De Landaeta et al. (2012), quienes reportaron valores de $13,6 \%$, para productos comercializados, en Argentina; igualmente, García et al. (2005) obtuvieron valores de 5,15\%, para salchichas elaboradas con carne

Tabla 3. Parámetros fisicoquímicos de las salchichas elaboradas.

\begin{tabular}{|l|c|c|c|c|}
\hline Parámetro analizado & $\begin{array}{c}\text { Salchicha de carne } \\
\text { de cerdo 100\% ha- } \\
\text { rina de trigo }\end{array}$ & $\begin{array}{c}\text { Salchicha de carne de } \\
\text { cerdo 65\% harina de } \\
\text { trigo y 35\% quitosano }\end{array}$ & $\begin{array}{c}\text { Salchicha de filete de } \\
\text { tilapia 100\% harina de } \\
\text { trigo }\end{array}$ & $\begin{array}{c}\text { Salchicha de filete de } \\
\text { tilapia 65\% harina de } \\
\text { trigo y 35\% quitosano }\end{array}$ \\
\hline Humedad, \% & $63,29 \pm 0,45^{\mathrm{a}}$ & $66,15 \pm 0,27^{\mathrm{a}}$ & $68,96 \pm 0,73^{\mathrm{b}}$ & $69,81 \pm 0,57^{\mathrm{b}}$ \\
\hline Proteína total (N x6,25), \% & $12,18 \pm 0,62^{\mathrm{a}}$ & $12,24 \pm 0,75^{\mathrm{a}}$ & $10,06 \pm 0,71^{\mathrm{b}}$ & $10,48 \pm 0,92^{\mathrm{b}}$ \\
\hline Grasa total, \% & $11,02 \pm 0,34^{\mathrm{a}}$ & $10,91 \pm 0,66^{\mathrm{a}}$ & $4,79 \pm 0,95^{\mathrm{b}}$ & $5,61 \pm 0,83^{\mathrm{b}}$ \\
\hline Cenizas, \% & $4,12 \pm 0,57^{\mathrm{a}}$ & $4,34 \pm 0,39^{\mathrm{a}}$ & $5,99 \pm 0,88^{\mathrm{a}}$ & $5,78 \pm 0,68^{\mathrm{a}}$ \\
\hline Carbohidratos totales, \% & $9,39 \pm 0,45^{\mathrm{a}}$ & $6,36 \pm 0,52^{\mathrm{a}}$ & $10,20 \pm 0,82^{\mathrm{b}}$ & $8,32 \pm 0,75^{\mathrm{b}}$ \\
\hline Caloría totales, Kcal/100g & $183,12 \pm 1,15^{\mathrm{a}}$ & $172,61 \pm 1,61^{\mathrm{a}}$ & $126,14 \pm 0,99^{\mathrm{b}}$ & $125,69 \pm 1,14^{\mathrm{b}}$ \\
\hline Nitritos, ppm & $136,38 \pm 0,27^{\mathrm{a}}$ & $137,37 \pm 0,36^{\mathrm{a}}$ & $199,89 \pm 0,15^{\mathrm{b}}$ & $202,18 \pm 0,21^{\mathrm{b}}$ \\
\hline Almidón, \% & $1,62 \pm 0,33^{\mathrm{a}}$ & $1,54 \pm 0,23^{\mathrm{a}}$ & $0,98 \pm 0,64^{\mathrm{a}}$ & $0,94 \pm 0,41^{\mathrm{a}}$ \\
\hline
\end{tabular}

Dentro de una fila los valores con diferente letra representa diferencias significativas $p<0,05$. 
de atún, valores similares a los encontrados en la presente investigación. El análisis relacionado con el contenido de grasas mostró que las salchichas elaboradas, se hallan dentro de los parámetros estipulados por la Norma Técnica Colombiana NTC 1325, que permite, para productos Premium, un contenido no superior a $28 \%$, de grasa total.

Respecto a los carbohidratos, su contenido fue mayor en las salchichas elaboradas a partir de filetes de tilapia. Para los cuatro tipos de salchichas, el contenido fue ligeramente superior a lo permitido por la NTC 1325, que permite un máximo de 5\%, para productos Premium. Teniendo en cuenta que la adición de harina de trigo y quitosano fue igual para cada grupo de salchichas, se recomienda revisar la formulación, para adecuarla a lo estipulado por la norma.

Respecto al aporte de calorías totales, las salchichas elaboradas a base de carne de cerdo registraron los valores más altos. Igualmente, en los productos elaborados, se observa un mayor valor que en las carnes materia prima, debido a la grasa que contienen las salchichas, siendo mayor su contenido en la carne de cerdo que en los filetes de tilapia. Lo anterior está acorde con los datos reportados por el Departamento de Agricultura de los Estados Unidos (USDA, 2015), con $1,97 \%$ de grasa, para filete de tilapia y $9,21 \%$, para carne fresca de cerdo, así como también, con los reportados -para filete de tilapia-, por Hernández-Sánchez \& Aguilera-Morales (2012), de 0,79 a $8,5 \%$.
La cantidad de nitritos (ppm) varía entre los grupos de salchichas, ajustándose ambos a lo estipulado en la Norma Técnica Colombiana NTC 1325.

Es importante resaltar que, estadísticamente, la inclusión de quitosano no influyó en los resultados de los parámetros fisicoquímicos evaluados para cada grupo de salchichas elaboradas; sin embargo, al analizar dentro de cada grupo las salchichas con $100 \%$ de inclusión de harina de trigo y aquellas con adición de 35\% de quitosano, sí se observan diferencias en los parámetros analizados.

Parámetros microbiológicos. Los resultados del análisis microbiológico (Tabla 4) muestran que, tanto las salchichas de carne de cerdo como las elaboradas a base de filete de tilapia, cumplen con los requisitos establecidos por la Normas Técnicas Colombianas NTC 1325 (2008) y NTC 4519 (2009).

La carne no es una materia prima estéril y los condimentos y las especias pueden contener microorganismos, que no mueren en el proceso de escaldado, ocasionando una aceleración en el deterioro de los productos finales. Los resultados obtenidos para los cuatro tipos de salchichas, se ubicaron dentro de lo permitido por las normas 1325 y 4519, establecidas por el ICONTEC NTC 1325 (2008) y NTC 4519 (2009), lo cual, permite asegurar una buena calidad e inocuidad de los mismos. Los resultados derivados de esta investigación

Tabla 4. Análisis microbiológicos para los productos elaborados.

\begin{tabular}{|c|c|c|c|c|c|c|}
\hline $\begin{array}{c}\text { Parámetros } \\
\text { analizado }\end{array}$ & Método & $\begin{array}{c}\text { Salchicha de } \\
\text { carne de cerdo } \\
100 \% \text { harina de } \\
\text { trigo }\end{array}$ & $\begin{array}{c}\text { Salchicha de } \\
\text { carne de cerdo } \\
65 \% \text { harina de } \\
\text { trigo y } 35 \% \\
\text { quitosano }\end{array}$ & $\begin{array}{c}\text { Salchicha de } \\
\text { filete de tilapia } \\
100 \% \text { harina de } \\
\text { trigo }\end{array}$ & $\begin{array}{c}\text { Salchicha de } \\
\text { filete de tilapia } \\
65 \% \text { harina de } \\
\text { trigo y 35\% } \\
\text { quitosano }\end{array}$ & Valor admisible \\
\hline $\begin{array}{c}\text { Aerobios } \\
\text { mesófilos UFC g }\end{array}$ & NTC 4519 & $<10$ & $<10$ & 60 & 50 & $<100.000$ \\
\hline $\begin{array}{c}\text { Coliformes totales } \\
\text { UFC g-1 }\end{array}$ & NTC 4458 & $<10$ & $<10$ & $<10$ & $<10$ & 500 Max. \\
\hline $\begin{array}{c}\text { Escherichia coli } \\
\text { UFC g-1 }\end{array}$ & NTC 4458 & $<10$ & $<10$ & $<10$ & $<10$ & $<10$ \\
\hline $\begin{array}{c}\text { Esporas } \\
\text { Clostridium Sulfit } \\
\text { Reductor UFC } \text {-1 }^{-1}\end{array}$ & INVIMA & $<10$ & $<10$ & $<10$ & $<10$ & 100 Max. \\
\hline $\begin{array}{c}\text { Salmonella } \\
\text { en 25g }\end{array}$ & NTC 4574 & Ausencia & Ausencia & Ausencia & Ausencia & Ausencia \\
\hline $\begin{array}{c}\text { Estafilococo } \\
\text { coagulasa } \\
\text { positiva UFC g }\end{array}$ & NTC 4779 & $<100$ & $<100$ & $<100$ & $<100$ & $<100$ \\
\hline
\end{tabular}


concuerdan con los reportados por García et al. (2005) y por Izquierdo et al. (2007), quienes, en sus respectivos trabajos, sugieren que el bajo conteo microbiológico, se debe al uso de materia fresca y, su buen manejo sanitario, a la alta temperatura en la cocción, al rápido enfriamiento del producto y al uso de envolturas impermeables. A pesar que el resultado de Escherichia coli en los productos elaborados fue menor de 10 UFC $\mathrm{g}^{-1}$, lo que coincide con el límite permisible por las normas colombianas, la presencia de bacterias coliformes en los alimentos no significa, necesariamente, que hubo una contaminación o que existen patógenos entéricos presentes (Castillo \& Andino, 2010).

Parámetros sensoriales. Se tuvieron en cuenta los resultados obtenidos, dentro de la escala hedónica de "me gusta muchísimo", "me gusta mucho" y "me gusta ligeramente", para los parámetros sabor, olor, color, textura y opinión general (Figura 1).

La media de los datos recolectados entre los jurados osciló alrededor de "me gusta mucho", en los atributos sabor y olor. Respecto al color de las muestras presentadas, los datos variaron entre "me gusta mucho" y "me gusta ligeramente", citando comentarios referentes a color muy pálido, en comparación con salchichas comerciales; con relación a la textura y a la opinión general, las respuestas se registraron entre "me gusta mucho" y "me gusta ligeramente". Para todos los atributos sensoriales, a excepción del color, las opiniones de más del $50 \%$ de los jueces se encontraron en los niveles "me gusta muchísimo" y "me gusta mucho", lo cual,

\author{
$\approx$ Salchichas carne de cerdo $100 \%$ harina de trigo \\ - Salchichas carne de cerdo $65 \%$ harina de trigo, $35 \%$ quitosano \\ - Salchichas filete de tilapia $100 \%$ harina de trigo \\ - Salchichas filete de tilapia $65 \%$ harina de trigo, $35 \%$ quitosano
}

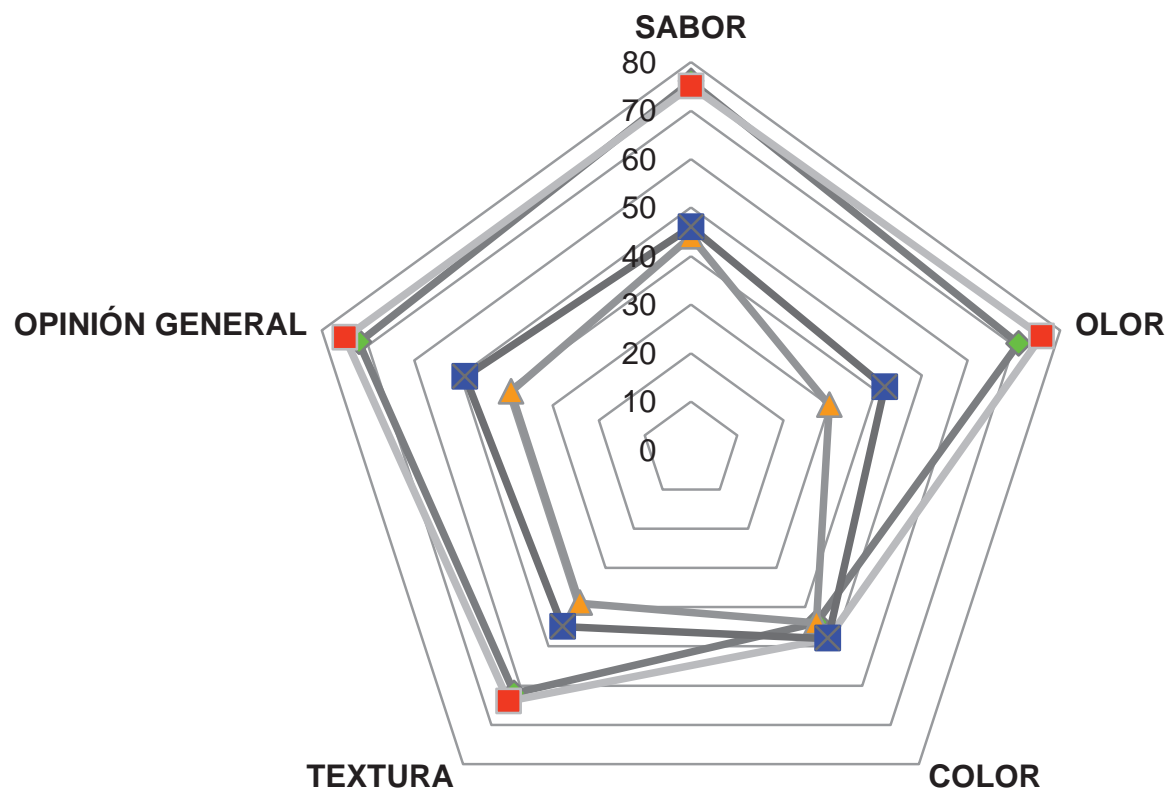

Los valores de la escala 0 a 80 representan las respuestas de los evaluadores, en los 3 puntos significativos de la escala hedónica.

Figura 1. Parámetros sensoriales para los productos elaborados. 
permitió concluir que se trató de salchichas de muy buena aceptación. La adición de quitosano, como agente extensor, en una cantidad de $35 \%$ de sustitución de harina de trigo, no afecta los aspectos sensoriales de las salchichas elaboradas con carne de cerdo; ésto se corroboró con el análisis estadístico, al notar que la mayor frecuencia expresada entre las personas encuestadas con respecto a sabor, olor, textura y opinión general de las salchichas elaboradas con carne de cerdo fue "me gusta mucho"; respecto al color, la mayor frecuencia fue "ni me gusta ni me disgusta". A las salchichas no se les adicionó colorante suplementario, resultando las salchichas con su color natural, que difiere con las salchichas comerciales, habituales para los jueces evaluadores.

Para las salchichas elaboradas a partir de filete de tilapia, la frecuencia de los datos obtenidos en las encuestas, se encontró entre "me gusta ligeramente", "ni me gusta, ni me disgusta" y "me disgusta ligeramente", para todos los atributos elaborados, que presentaron opinión por encima del $50 \%$ de los evaluadores, en estos tres puntos de la escala hedónica y ello, se puede deber al poco conocimiento que se tiene de este tipo de productos, que no son comerciales en Colombia y no se tiene la referencia para su consumo. Con relación al atributo color, se tuvieron las mismas apreciaciones que en el caso de las salchichas de carne de cerdo y, esto igualmente se explica, por la no adición de colorantes artificiales, a los productos elaborados.

Finalmente, las dos muestras con 35\% de adición de quitosano fueron sometidos a una prueba de aceptación y de preferencia, por parte de los 77 jueces evaluadores, la cual, mostró que de las dos salchichas, la de mayor aceptación fue la elaborada a partir de carne de cerdo. En el análisis estadístico ANOVA, se nota una preferencia por las salchichas elaboradas con carne de cerdo, por 61 de los evaluadores, mientras que la aceptación por las salchichas a base de filete de tilapia fue de 16 opiniones positivas, mostrando una diferencia significativa entre las dos muestras.

Se obtuvieron dos tipos de salchichas: uno, elaborado a base de carne de cerdo y, el otro, de filete de tilapia, ambos con adición de quitosano, en una sustitución del $35 \%$ del total de harinas utilizada en su fabricación, como elemento extensor. De los dos productos fabricados, la salchicha de carne de cerdo tuvo la mejor aceptación sensorial, mientras que la de filete de pescado, no fue de gran agrado, debido, probablemente, al desconocimiento en el medio de este producto no tradicional.

Conflicto de intereses. El manuscrito fue preparado y revisado con la participación de todos los autores, quienes declaramos que no existe conflicto de intereses que ponga en riesgo la validez de los resultados presentados. Financiación: Este estudio fue financiado por la Universidad Nacional de
Colombia - sede Palmira, dentro del Programa Nacional de Semilleros de Investigación, Creación e Innovación, de la Universidad Nacional de Colombia 2013 - 2015.

\section{BIBLIOGRAFÍA}

1. ABDEL-MOHSEN, A.; ABDEL-RAHMAN, R.; HRDINA, R.; IMRAMOVSKÝ, A.; BURGERT, L.; ALY, A. 2012. Antibacterial cotton fabrics treated with core-shell nanoparticles. Int. J. Biol. Macromol. 50(5):12451253.

2. ABDEL-MOHSEN, A.; ABDEL-RAHMAN, R.; FOUDA, M.; VOJTOVA, L.; UHROVA, L.; HASSAN, A.; ALDEYAB, S.; EL-SHAMY, I.; JANCAR, J. 2014. Preparation, characterization and cytotoxicity of schizophyllan/silver nanoparticle composite. Carbohydr. Polymers. 102:238-245.

3. ANDÚJAR, G.; GUERRA, A.; SANTOS, R. 2000. La utilización de extensores cárnicos. Experiencias de la industria cárnica cubana. Instituto de investigaciones para la industria alimenticia. Disponible desde Internet en: http://www.rlc.fao.org/prior/segalim/pdf/ extensor.pdf (con acceso 15/09/2014).

4. AOAC. 2011. Official Method in Meat and Meat Products. Gaithersburg, MD: AOAC International.

5. AUTORIDAD NACIONAL DE ACUICULTURA Y PESCA -AUNAP-. 2014. La pesca y la Acuicultura en Colombia 2014. Documento de compilación de información. 26p.

6. BLANNO, M. 2006. Extensores cárnicos: consideraciones de funcionalidad y valor nutricional. Mundo Lácteo y Cárnico. (México). 1:9-13.

7. CASTILLO, Y.; ANDINO, F. 2010. Curso Microbiología de los alimentos: Un enfoque práctico para la inocuidad alimentaria. Universidad Nacional de Ingeniería UNI - Estelí. Nicaragua, 61p.

8. DE LANDAETA, M.; PIGHIN, A.; MARCHESICH, C.; CABRERA, M.; MARCHINI, M. 2012. Composición centesimal y contenido de minerales en comidas rápidas: hamburguesas y salchichas de viena de primeras marcas crudas y cocidas. Diaeta. (Argentina). 30(140):18-24.

9. GARCÍA, A.; IZQUIERDO, P.; UZCÁTEGUI, S.; FARÍA, J.; ALLARA, M. 2005. Formulación de salchichas con atún y carne: Vida útil y aceptabilidad. Rev. Cient. FCV-LUZ. (Venezuela). 15(3):272-278. 
10. GEORGANTELIS, D.; BLEKAS, G.; KATIKOU, P.; AMBROSIADIS, I.; FLETOURIS, D. 2007. Effect of rosemary extract, chitosan and $\alpha$-tocopherol on lipid oxidation and colour stability during frozen storage of beef burgers. Meat Sci. (USA). 75(2):256-264.

11. GÜEMES, N. 2007. Utilización de los derivados de los cereales y leguminosas en la elaboración de productos cárnicos. Nacameh. (México). 1(2):110-117.

12. HERNÁNDEZ-SÁNCHEZ, F; AGUILERA-MORALES, M. 2012. Nutritional richness and importance of the consumption of tilapia in the Papaloapan Region. Rev. Electr. Vet. (España). 13(6):1-12.

13. HLEAP, J.; VELASCO, V. 2010a. Análisis de las propiedades de textura durante el almacenamiento de salchichas elaboradas a partir de tilapia roja (Oreochromis sp.). Biotecn. Sector Agrop. Agroind. (Colombia). 8(2):46-56.

14. HLEAP, I.; GUTIÉRREZ, A.; RIVERA, L. 2010b. Análisis microbiológico y sensorial de productos elaborados a partir de surimi de carduma (Cetengraulis mysticetus) y plumuda (Opistonema spp.). Biotecn. Sector Agrop. Agroind. 8(2):57-65.

15. ICONTEC. 2007. Normas Técnicas Colombianas 4458, 4574 y 4779 . Microbiología de alimentos y alimentos para animales. $1^{\text {a }}$ Act. Bogotá.

16. ICONTEC. 2008. Norma Técnica Colombiana 1325. Industrias alimentarias. Productos cárnicos procesados no enlatados. $5^{\text {a }}$ Act. Bogotá.

17. ICONTEC. 2009. Norma Técnica Colombiana 4519. Microbiología de los alimentos para consumo humano y animal. $1^{\mathrm{a}}$ Act. Bogotá.

18. INSTITUTO NACIONAL DE VIGILANCIA DE MEDICAMENTOS Y ALIMENTOS -INVIMA-. 1998. Manual de técnicas de análisis para control de calidad microbiológico de alimentos para consumo humano. Bogotá, Colombia. 22p.

19. INTARASIRISAWAT, R.; BENJAKUL, S.; VISESSANGUAN, W.; WU, J. 2014. Effect of skipjack roe protein hydrolysate on properties and oxidative stability of fish emulsion sausage. LWT - Food Sci. Technol. 58(1):280-286.

20. IZQUIERDO, P.; GARCÍA, A.; ALLARA, M.; ROJAS, E.;
TORRES, G.; GONZÁLEZ, P. 2007. Análisis proximal, microbiológico y evaluación sensorial de salchichas elaboradas a base de cachama negra (Colossoma macropomum). Rev. Cient. FCV-LUZ. 17(3):294300.

21. JEON, Y.; KAMIL, J.; SHAHIDI, F. 2002. Chitosan as an edible invisible film for quality preservation of herring and Atlantic cod. J. Agr. Food Chem. (USA). 50(18):5167-5178.

22. KRAJEWSKA, B. 2004. Application of chitin - and chitosan-based materials for enzyme immobilizations: a review. Enzyme Microbial Technol. 35(2-3):126-139.

23. MAHDY, S.; EL-KALYOUBI, M.; KHALAF, M.; EL-RAZIK, A. 2013. Physicochemical, functional, antioxidant and antibacterial properties of chitosan extracted 54 from shrimp wastes by microwave technique. Ann. Agr. Sci. (Egypt). 58(1):33-41.

24. MÁRMOL, Z.; PÁEZ, G.; RINCÓN, M.; ARAUJO, K.; AIELLO, C.; CHANDLER, C.; GUTIÉRREZ, E. 2011. Quitina y quitosano polímeros amigables. Una revisión de sus aplicaciones. Rev. Tecnocient. URU. (Venezuela). 1:53-58.

25. NO, H.; MEYERS, S.; PRINYAWIWATKUL, W.; XU, Z. 2007. Applications of chitosan for improvement of quality and shelf life of foods: a review. J. Food Sc. 72(5):87-100.

26. O'FLYNN, C.; CRUZ-ROMERO, M.: TROY, D.; MULLEN, A.; KERRY, J. 2014. The application of high-pressure treatment in the reduction of phosphate levels in breakfast sausages. Meat Sci. 96(1):633-639.

27. PRZEKORA, A.; GINALSKA, G. 2014. Biological properties of novel chitosan-based composites for medical application as bone substitute. Central Eur. J. Biol. 9(6):634-641.

28. RABEA, E.; BADAWY, M.; STEVENS, C.; SMAGGHE, G.; STEURBAUT, W. 2003. Chitosan as antimicrobial agent: applications and mode of action. Biomacromolecules. (United States). 4(6):1457-1465.

29. RAMYA, R.; VENKATESAN, J., KIM, S.; SUDHA, P. 2012. Biomedical applications of chitosan: an overview. J. Biomat. Tissue Enginee. (United States). 2(2):100111.

30. RINAUDO, M. 2006. Chitin and chitosan: properties and applications. Progr. Polymer Sci. 31(7):603-632. 
31. SAVADKOOHI, S.; HOOGENKAMP, H., SHAMSI, K.; FARAHNAKY, A. 2014. Color, sensory and textural attributes of beef frankfurter, beef ham and meatsausage containing tomato pomace. Meat Sci. 97(4):410-418.

32. SHAHIDI, F.; VIDANA, J.; JEON, Y. 1999. Food applications of chitin and chitosans. Trends Food Sci. Technol. 10(2):37-51.

33. THARANATHAN, R.; KITTUR, F. 2003. Chitin - the undisputed biomolecule of great potential. Crit. Rev. Food Sci. Nutr. (USA). 43(1):61-87.

34. UNITED STATES DEPARTMENT OF AGRICULTURE USDA-. 2015. National Nutrient Database for Standard Reference. Disponible desde Internet en: http:// fnic.nal.usda.gov/food-composition (con acceso 17/06/2015).

35. VELÁZQUEZ-MOREIRA, A.; GUERRERO, J. 2014. Algunas investigaciones recientes en recubrimientos comestibles aplicados en alimentos. Temas Selectos Ing. Alimentos. (México). 8(2):5-12.
36. YANG, H.; CHOI, S.; JEON, J.; PARK, G.; JOO, S. 2007. Textural and sensory properties of low fat pork sausages with added hydrated oatmeal and tofu as texturemodifying agents. Meat Sc. 75(2):283-289.

37. ZENG, D.; LUO, X.; TU, R. 2012. Application of bioactive coatings based on chitosan for soybean seed protection. Int. J. Carbohydr. Chem. (United States). Article ID 104565, 5p.

38. ZHANG, Y.; ZHANG, X.; DING, R.; ZHANG, J.; LIU, J. 2011. Determination of the degree of deacetylation of chitosan by potentiometric titration preceded by enzymatic pretreatment. Carbohydr. Polymers. 83(2):813-817.

Recibido: Mayo 6 de 2015

Aceptado: Octubre 13 de 2015

Cómo citar:

Hleap, J.I.; Cardona, L.; Agudelo, J.; Gómez, A. 2015. Parámetros fisicoquímicos, microbiológicos y sensoriales de salchichas elaboradas con inclusión de quitosano. Rev. U.D.C.A Act. \& Div. Cient. 18(2): 455-464. 